Vol 13, Issue 5, 2020

\title{
STUDY OF PRESCRIBING PATTERN OF ANTIMICROBIAL AGENTS IN MEDICINE INTENSIVE CARE UNIT OF A TERTIARY CARE HOSPITAL
}

\author{
DUDHE BG, CHAKRAVORTY ASHISH D*, MANKAR NN, PISE HN
}

Department of Pharmacology, Government Medical College, Nagpur, Maharashtra, India. Email: dr_aashish77@rediffmail.com

Received: 27 December 2019, Revised and Accepted: 20 March 2020

ABSTRACT

Objective: The objective of the study was to assess the current use of antimicrobial agents (AMAs) and to assess prescription writing as per the WHO guidelines in the medicine intensive care unit (MICU).

Methods: The present study was conducted on admitted patients in MICU of a tertiary care hospital. The demographic and clinical treatment data of patients were collected in specially designed pro forma from the case record form.

Results: Of 400 patients enrolled in the study, 55.50\% were male and 44.50\% were female. A common indication of AMAs use was infection (61.75\%), symptomatic (21.50\%), and prophylactic (10.50\%) use. In $65 \%$ patients, antimicrobial therapy was considered to be rational. The majority of patients have good recovery (61\%). Polypharmacy was seen in $89.75 \%$ of patients. The average number of drugs prescribed per patient was $8.84 \pm 2.55 .1 .90 \pm 1.20$ was average number of AMAs per patient. Commonest AMAs prescribed are ceftriaxone (27.64\%), metronidazole, and amoxicillin+clavulanic acid.

Conclusion: This study visibly highlights the practice of polypharmacy, low uses of generic drugs, high usage of antibiotic, and injection. Cephalosporins are the most prescribed AMAs in the ICUs. Remedy of this situation requires regulation, education, awareness, compliance with protocol, and guidelines of AMAs use.

Keywords: Antimicrobial agents, Prescription, Drug utilization, Intensive care unit.

(C) 2020 The Authors. Published by Innovare Academic Sciences Pvt Ltd. This is an open access article under the CC BY license (http://creativecommons. org/licenses/by/4. 0/) DOI: http://dx.doi.org/10.22159/ajpcr.2020.v13i5.36720

\section{INTRODUCTION}

Antibiotics are the substances that kill or inhibit the growth of microorganisms and are used for the treatment or prevention of different types of local and systemic infections [1]. Antibiotics help in reducing the burden of mortality and morbidity caused by infectious diseases [2]. Most of the patients in medicine intensive care unit (MICU) often suffer from severe illnesses, multiple organ dysfunction, and coexisting medical disorders. In such situation, multiple drugs are prescribed routinely. A major portion of drugs prescribed to the patients admitted in MICU consist of antimicrobial agents (AMAs) [3].

Unnecessary poly-pharmacy, high use of drugs with unproven efficacy, and irrational antibiotic usage are some of the recognized drug prescription issues in developing countries. These problems lead to increased health-care utilization, morbidity, mortality, adverse drug events, and drug resistance [4]. Inappropriate antibiotic prescribing is a grave danger worldwide, as many organisms have become resistant to commonly used antibiotics [2,5]. Although there are guidelines and appeal by Government and professional medical societies to decrease antibiotic prescribing for inappropriate indications, there has been a slight improvement in prescribing patterns [6,7]. Furthermore, information regarding antibiotic prescribing trends that can direct stewardship efforts remains sparse in developing countries.

A physician needs to possess a skill of prescribing drugs. This skill requires to be assessed and refined time to time. It reflects the physician's knowledge of medicine, his accuracy in diagnosis and attitude toward choosing the rational treatment [8]. The study of prescribing patterns will provide baseline data to suggest modifications in guidelines of prescription writing and antibiotic stewardship program. This may help in a great deal to reduce bacterial resistance.
With this state of affairs of antimicrobials, we planned to conduct a study with the aim to assess the prescribing pattern of AMAs in MICU of a teaching hospital in Central India. The primary objectives are to assess the current use of AMAs in the MICU and to assess prescription writing as per the WHO guidelines.

\section{METHODS}

The present study was conducted on admitted patients in MICU of a tertiary care hospital. The study was conducted after obtaining due approval from the Institute Ethics Committee. Informed consent was obtained from each patient or relatives of the patient admitted in MICU. The demographic and clinical treatment data of 400 patients were collected in specially designed pro forma from the case record form. Baseline demographic variables were recorded. The prescription data of all patients at admission into the MICU were noted with regard to antibiotic prescription and the class of antibiotics. The antibiotic prescription pattern was analyzed until the patient was discharged from MICU.

\section{Inclusion criteria}

The following criteria were included in the study:

- Patients admitted in MICU

- Patients or relatives willing to give written informed consent.

\section{Exclusion criteria}

The following criteria were excluded from the study:

- Patients not ready to give written informed consent.

- Patients not willing to be followed up, if required.

- Incomplete data and patients who stayed for $<24 \mathrm{~h}$ in MICU.

Indication for the antimicrobial use

The following groups were essentially defined by the physician according to the way they treated the patients $[9,10]$. 
1. If clinical and/or laboratory data gave evidence of infection, the infection was considered as the indication

2. If there was no evidence of infection and the agent was employed to prevent infection (e.g., catheterization), the therapy was considered as prophylactic

3. If no evidence of prophylaxis could be found and records showed the same symptoms being treated, for example, antimicrobial use in the treatment of fever in the absence of specifically suspected infection, the indication was considered as symptomatic.

\section{Rationality $[9,10]$}

a. If the antimicrobial use and its dose, route of administration, frequency, and duration of use were considered appropriate for infection, the therapy was considered rational

b. If the antimicrobial was used without indication, prophylaxis under circumstances of unproven efficacy or by clearly inappropriate route, dose, or preparation for that indication, therapy was considered irrational

c. When insufficient clinical or laboratory data were present to help the therapy to be classified as clearly rational or irrational, for example, the patient of congestive heart failure having cough but do not know that cough is due to CHF or infection then treatment with AMA considered questionable, therapy was considered questionable.

\section{Statistical analysis}

Data were analyzed using descriptive statistics. Statistical software Graph Pad Prism was used for the analysis of data and Microsoft Word and Excel to generate graphs and tables.

\section{RESULTS}

During the study period total, 400 patients were enrolled, consisting $222(55.50 \%)$ male and $178(44.50 \%)$ females patient. 1.25 was male to female ratio. Middle age male was the most common age group among all admitted patients. Patients above 40 years were 293 (73.25\%). The mean age of patients was $52.01 \pm 15.33$ years (Table 1 ).

As shown in Fig. 1, the indication for antimicrobial use in which common indication was infection (61.75\%) followed by prophylactic use $(21.50 \%)$ and symptomatic use (10.50\%). Fig. 2 shows the evaluation of antimicrobial therapy according to its use, wherein $65.00 \%$ patients the antimicrobial therapy was considered rational, in $23.75 \%$ patients, therapy was irrational, while in $11.25 \%$ patients, it was questionable. In this study, a good prognosis was seen in $61 \%$ of patients and mortality rate was found to be $13.50 \%$. The majority of the prescriptions were properly signed (52.25\%) followed by improperly signed (28\%) and absence of signature $(19.75 \%)$.

As depicted in Table 2, 2.89.75\% of patients received more than five drugs per day, $66.75 \%$ patients received 6-10 drugs per day, and $23 \%$ received $11-15$ drugs. It was observed that the maximum number of drugs prescribed to a single patient was 15 . The average number of drugs prescribed per patient was $8.84 \pm 2.55$. Fig. 3 depicts 158 (39.50\%) patients received only one AMA, 24.25\% received two AMAs, and $30.50 \%$ received 3-6 AMAs. The average number of AMAs per patient was $1.90 \pm 1.20$. Only $20.20 \%$ of AMAs were prescribed by generic name while majority by brand name. The most common dosage form used for AMA was injection (76.08\%) followed by tablet $(19.35 \%)$ and nebulization $(4.57 \%)$

The commonly prescribed antimicrobial drug classes were cephalosporins (31.13\%) and penicillins (18.03\%). Ceftriaxone $(27.64 \%)$ was the commonest AMA followed by metronidazole $(17.79 \%)$ to be prescribed. Prescribing frequency of AMAs among patients in the MICU has been detailed in Table 3. A total of 175 (20.03\%) antimicrobial fixed drug combinations (FDC) were used among 400 patients. Amoxicillin+clavulanic acid (11.30\%) was the most common FDC noticed. The number of patients who were prescribed antimicrobial FDCs is shown in Table 4.

\section{DISCUSSION}

A prescription by a doctor may be taken as a reflection of the doctor's attitude toward the disease and the role of the drug in treatment. It provides valuable insight into the nature of the health-care delivery system of the country [11]. Quality-of-life can be raised by enhancing standards of medical treatment at all levels. Setting regulations and assessing the quality of health care through performance review and audits should become a part of everyday clinical practice [12].

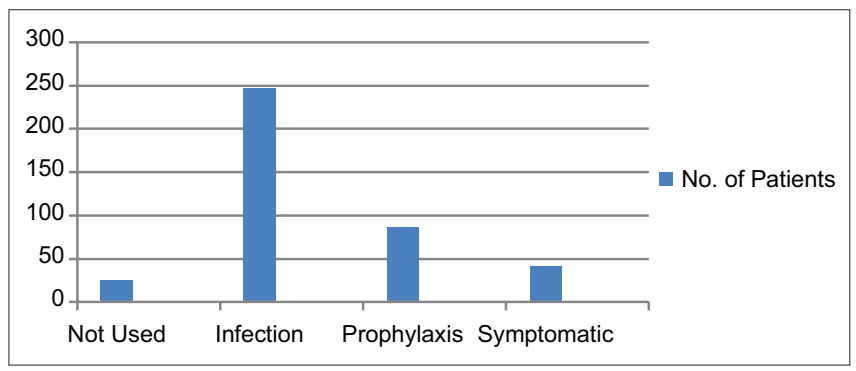

Fig. 1: Indication for antimicrobial use

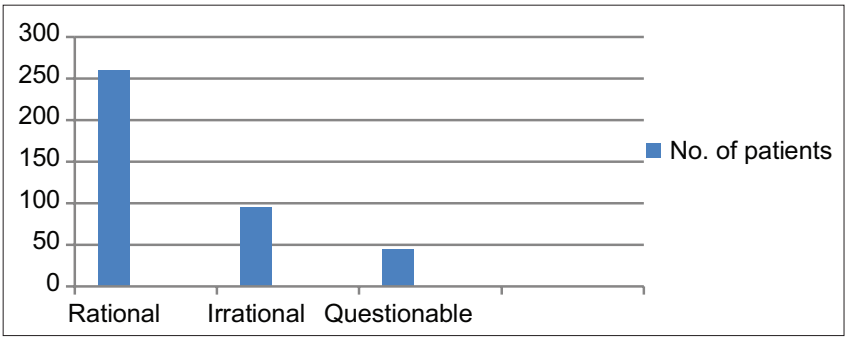

Fig. 2: Evaluation of antimicrobial therapy according to use

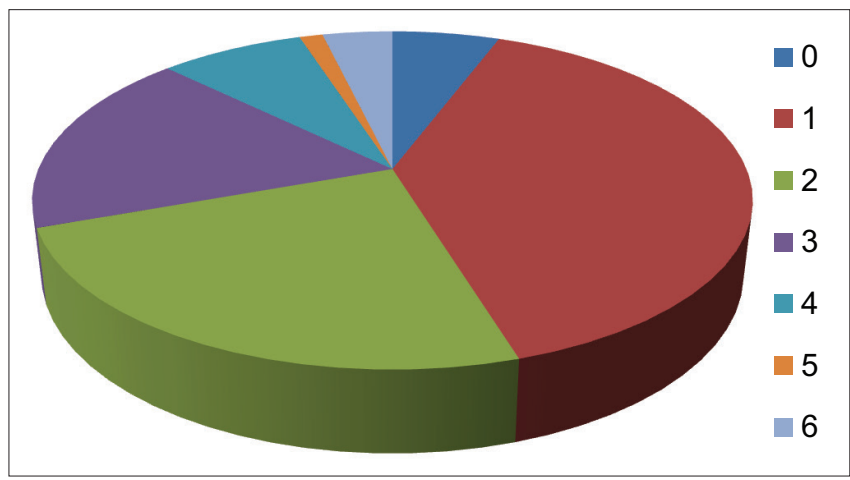

Fig. 3: Total number of antimicrobial agents per patient

Table 1: Age- and sex-wise distribution of the patients

\begin{tabular}{lll}
\hline Parameters & No. of patients & Percentage \\
\hline Age (years) & & \\
$11-20$ & 08 & 2.00 \\
$21-30$ & 33 & 8.25 \\
$31-40$ & 66 & 16.50 \\
$41-50$ & 74 & 18.50 \\
$51-60$ & 92 & 23.00 \\
$61-70$ & 84 & 21.00 \\
$71-80$ & 30 & 07.50 \\
$81-90$ & 13 & 03.25 \\
Sex & & \\
Male & 222 & 55.50 \\
Female & 178 & 44.50 \\
\hline
\end{tabular}


Table 2: Total number of drugs prescribed per patients

\begin{tabular}{llll}
\hline S. No. & $\begin{array}{l}\text { Total No. of drugs } \\
\text { prescribed }\end{array}$ & No. of patients & $\begin{array}{l}\text { Percentage of } \\
\text { patients }\end{array}$ \\
\hline 1. & 1 & 00 & 00 \\
2. & 2 & 3 & 00.75 \\
3. & 3 & 2 & 00.50 \\
4. & 4 & 8 & 02.00 \\
5. & 5 & 28 & 07.00 \\
6. & 6 & 43 & 10.75 \\
7. & 7 & 48 & 12.00 \\
8. & 8 & 51 & 12.75 \\
9. & 9 & 43 & 10.75 \\
10. & 10 & 82 & 20.50 \\
11. & 11 & 31 & 07.75 \\
12. & 12 & 38 & 09.50 \\
13. & 13 & 10 & 02.50 \\
14. & 14 & 3 & 00.75 \\
15. & 15 & 10 & 02.50 \\
\hline
\end{tabular}

Table 3: Commonly prescribed antimicrobial agents

\begin{tabular}{llll}
\hline S. No. & Name of drug & No. of patients & Percentage \\
\hline 1. & Ceftriaxone & 230 & 27.64 \\
2. & Metronidazole & 148 & 17.79 \\
3. & Amoxicillin+Clavulanic acid & 94 & 11.30 \\
4. & Ofloxacin & 61 & 7.33 \\
5. & Azithromycin & 53 & 6.37 \\
6. & Piperacillin+Tazobactam & 47 & 5.64 \\
7. & Levofloxacin & 30 & 3.60 \\
8. & Cefixime & 29 & 3.48 \\
9. & Doxycycline & 23 & 2.76 \\
10. & Artesunate & 21 & 2.52 \\
11. & Ofloxacin+Ornidazole & 19 & 2.28 \\
12. & Meropenem & 18 & 2.16 \\
13. & Rifaximine & 16 & 1.92 \\
14. & Ceftriaxone+Sulbactam & 15 & 1.80 \\
15. & Amikacin & 13 & 1.56 \\
16. & Amoxicillin & 09 & 1.08 \\
17. & Acyclovir & 3 & 0.36 \\
18. & Chloroquine & 2 & 0.24 \\
19. & Moxifloxacin & 1 & 0.12 \\
\hline
\end{tabular}

Table 4. Number of patients prescribed antimicrobial drugs in fixed-dose combination

\begin{tabular}{llll}
\hline S. No. & $\begin{array}{l}\text { Antimicrobial agents in } \\
\text { fixed-dose combinations }\end{array}$ & No. of patients & Percentage \\
\hline 1. & Amoxicillin+Clavulanic acid & 94 & 11.3 \\
2. & Piperacillin+Tazobactam & 47 & 5.65 \\
3. & Ofloxacin+Ornidazole & 19 & 2.28 \\
4. & Ceftriaxone+Sulbactam & 15 & 1.80 \\
\hline
\end{tabular}

In our study, male to female ratio was 1.25 , whereas in studies conducted by John et al. [13] and Naikwadi et al. [14], it was 1.9 and 1.4, respectively. Similar male preponderance has been shown in previous studies $[15,16]$. In contrast, a study done by Smythe et al. [17] showed an equal proportion of male and female admitted to the MICU. The difference in gender distribution was due to differences in admissions in MICU in an Indian setting. The probable reason for such a finding could be the higher male to female ratio in the central India. In the Indian scenario, it is noticed that female populations, especially the lower socioeconomic status, are reluctant to utilize health-care facilities, even if they are critically ill.

The mean age of the patients in MICU in this study was $52.01 \pm 15.33$ years, which is similar to a study by John et al. [13] and a study conducted in Srishyla et al. [18] as 50 years. In contrast, a study by Naikwadi et al. found mean age to be $34.16 \pm 14.13$ [14] while Mangrulkar et al. showed a mean age of $>60$ years $(62.2 \pm 16.24$ years $)$ [19]. The relatively high mean age in our study was due to the fact that the majority of the patients in this study were admitted due to cardiac, renal, neuronal, or pulmonary problems which are usually encountered at a relatively older age.

It has been found that the common indication for the use of AMAs in this study was infection (61.75\%) followed by prophylactic use $(21.50 \%)$ and symptomatic use $(10.50 \%)$. AMAs were not used in $6.25 \%$ of patients also. This is comparable to the result observed by Badar and Navale [9] wherein the indications were infection (64.9\%), symptomatic use (24\%), and prophylaxis (11\%) and to a study by Pandiamunian and Somasundaram [20], i.e., infection (63.4\%), prophylaxis $(23.9 \%)$, and symptomatic $(12.7 \%)$. In our study, evaluation of $65 \%$ of AMAs use was rational, $23.75 \%$ irrational, and $11.25 \%$ was questionable. These results are contradictory with a study by Badar and Navale [9], where $30.4 \%$ of AMAs evaluation was rational, 58.6\% irrational, and $11 \%$ questionable.

In this study, a good prognosis was seen in $61 \%$ of patients and the mortality rate was found to be $13.50 \%$. This is in accordance with the study done by Nikhilesh et al. [21] and a study done in Shankar et al. [22], wherein ICU mortality rate was found to be $12 \%$ and $15.4 \%$, respectively. However, many Indian studies reported very high MICU mortality rate as around $35 \%[13,23]$.

The average number of drugs prescribed to a patient in our study was $8.84 \pm 2.55$ which is comparable to a study conducted by Badar and Navale [9] and Pandiamunian and Somasundaram [20], wherein it was 7.5 and $10.4 \pm 2$, respectively. The number is lesser than that was observed by John et al. (11.6 \pm 2 drugs) and Smythe et al. $(12.1 \pm 7.6)[13,17]$ but is higher compared to a report from Nepal which recorded a mean of 5.1 \pm 2.7 drugs [22] and Alamchandani et al., wherein it was $4.12 \pm 1.49$ [24].

Polypharmacy is defined as concomitant use of five or more drugs and it could enhance drug-drug interactions and drug-related problems. Polypharmacy was seen in around $96.75 \%$ patients. It is difficult to treat patients in the ICU with multiple comorbidities with less number of drugs as they require multiple drugs for the treatment of the specific condition as well as for prophylaxis. It is essential to keep a balance between the number of drugs and effective pharmacotherapy. The mean number of drugs per prescription should be as low as possible since higher numbers increase the risk of drug interaction, risk of bacterial resistance, noncompliance, and cost of the treatment $[25,26]$.

In our study, patients received more than one AMA on a number of occasions (94.25\%). As these patients were suffering from mixed infections, they received one AMA for Gram positive, other for Gram negative and third one for anaerobic infection. This multidrug therapy is useful in decreasing the prevalence of drug resistance and total drug duration. A high percentage of patients were prescribed at least one antibiotic which was in accordance with a study conducted by Badar and Navale. (83\%), John et al. (83\%), and Shankar et al. (92\%) $[9,13,22]$ but was contrary to some studies where lesser AMAs were used such as Naikwadi et al. (70.85\%) and Curcio et al. (51\%) [15,27]. The mean number of AMAs per patient was $1.90 \pm 1.20$ which equals to that of studies conducted by Nikhilesh et al. (1.73) [21] and by Amit (1.74) [28].

In our study, drugs prescribed by generic name were only $20.20 \%$, which are very much comparable to the study by John et al. (30\%) and Pandiamunian and Somasundaram (29.21\%) [13,20]. Contradictory to these results, $79.18 \%$ drugs were prescribed by generic name in a study by Admane et al. and 100\% in Alharafsheh et al. [29,30] generic prescribing helps the hospital pharmacy to have better inventory control. Confusion among the pharmacists while dispensing, when prescribed by generic names, can also be reduced. Moreover, generic drugs are more cost-effective than the branded one [31]. 
In MICU, patients are always in critical condition, so they frequently receive drugs by the parental route to combat the emerging lifethreatening situation. The most common dosage form used for AMA was injection $(76.08 \%)$, which is similar to a study by Pandiamunian and Somasundaram (72.56\%) [20].

In our study, cephalosporins (31.13\%) and penicillins (18.03\%) were the commonly prescribed antimicrobial drug classes. Similar results were observed by Pandiamunian and Somasundaram [20], i.e., cephalosporins (35.6\%) and penicillins (21.3\%), whereas in a study by Jokandan and Jha [32], a different trend in the use of AMA was seen, i.e., cephalosporins $(22.03 \%)$, quinolone $(15.57 \%)$, antifungals $(14.36 \%)$, aminoglycosides (11.59\%), and penicillin (10.76\%).

Among the AMAs in our study, ceftriaxone was the commonest to be prescribed, followed by metronidazole, FDC of amoxicillin+clavulanic acid, ofloxacin, and azithromycin. Ceftriaxone was found to be used most commonly in some other studies also [9,33,34]. Usluer et al. [35] reported Biswal et al. [36] documented metronidazole as the most commonly prescribed AMA. Contradictory to above results ampicillin, amoxicillin, metronidazole, ciprofloxacin, and crystalline penicillin were the five most commonly prescribed AMAs in the study conducted by Shankar et al. [37] different trend in the use of AMA was seen in a study by John et al. [13] as cefoperazone (30\%), amikacin (28.9\%), and metronidazole $(28.6 \%)$. Another study by Naikwadi et al. and Nikhilesh et al. showed AMA use pattern of ceftriaxone, FDC of piperacillin+tazobactam, and metronidazole $[15,21]$. Another study by Maharani et al. showed yet another AMAs usage pattern with cefotaxime (37.6\%), metronidazole (19.9\%), azithromycin $(17.2 \%)$, and FDC of piperacillin+tazobactam (12.2\%) to be the common antibiotics used in MICU settings [38].

Cephalosporins are commonly prescribed because of their comparatively lower toxicity and broader spectrum activity. For several serious Gram-negative infections, cephalosporins in combination with aminoglycosides are frequently used due to synergistic activity and broader coverage of organisms. Recent studies done in this decade in our country documented that third-generation cephalosporins are the most commonly prescribed AMAs in the ICUs as well as in the outpatient clinics in India [20].

In this study, among total antimicrobials prescribed, $21.03 \%$ were FDC and amoxicillin+clavulanic acid (11.30\%) was the most common FDC noticed followed by piperacillin+tazobactam (5.65\%) and ofloxacin+ornidazole $(2.28 \%)$ which was in correspondence with a study conducted by Maharani et al., where most common combination used was amoxicillin+clavulanic, piperacillin+tazobactam, and amoxicillin+benzylpenicillin [38]. However, a study by John et al. [13] shows that cefoperazone+sulbactam combination was the most preferred FDC.

\section{LIMITATIONS OF STUDY}

There are certain limitations of this study as it was conducted in a single tertiary care setting and results cannot be generalized to the whole population in Central India. We looked at drug use patterns over a 4-month period only. The study was retrospective. Data on the scales used to grade the severity of illness of admitted patients such as acute physiology, age, and chronic health evaluation were not available in the case record. Hence, we were unable to correlate the drug prescribing patterns with the severity of patient illness.

\section{CONCLUSION}

This study clearly highlights the practice of polypharmacy, low uses of generic drugs, a very high usage of AMAs, injections, and cephalosporins being the most prescribed AMAs in the ICUs. Rational use of the drug is the foremost goal in writing a prescription. A multidisciplinary approach can be undertaken in the ICU set up. Pharmacists, microbiologists, infectious disease control specialists, and intensive care specialists can work together to lead a pathway for creating awareness and guidelines of rational antimicrobial pharmacotherapy. Future research should be aimed at determining the impact of incomplete prescriptions on a patient's health status and drug resistance. Remedy of this situation requires regulation, education, awareness, compliance to protocol, and guidelines of AMAs use. Overall, the scope for improving rational use of AMAs exists. The present study on antimicrobial drug prescribing patterns in MICU can provide a framework for continuous prescription audit.

\section{AUTHORS' CONTRIBUTIONS}

All the authors had contributed in conduct of the study and preparation of the manuscript.

\section{CONFLICTS OF INTEREST}

The authors declared no conflicts of interest regarding the research, authorship, and publication of this article.

\section{SOURCE OF FUNDING}

Self-funding.

\section{REFERENCES}

1. Leekha S, Terrell CL, Edson RS. General principles of antimicrobial therapy. Mayo Clin Proc 2011;86:156-67.

2. How to Investigate Antimicrobial Use in Hospitals: Selected Indicators; 2020. Available from: http://www.apps.who.int/medicinedocs/en $/ \mathrm{m} /$ abstract/Js21031en. [Last accessed on 2020 Jan 18].

3. Roca I, Akova M, Baquero F, Carlet J, Cavaleri M, Coenen S, et al. The global threat of antimicrobial resistance: Science for intervention. New Microbes New Infect 2015;6:22-9.

4. Isturiz RE, Carbon C. Antibiotic use in developing countries. Infect Control Hosp Epidemiol 2000;21:394-7.

5. Hashemi S, Nasrollah A, Rajabi M. Irrational antibiotic prescribing: A local issue or global concern? EXCLI J 2013;12:384-95.

6. Barnett ML, Linder JA. Antibiotic prescribing for adults with acute bronchitis in the United States, 1996-2010. JAMA 2014;311:2020-2.

7. Schmidt ML, Spencer MD, Davidson LE. Patient, provider, and practice characteristics associated with inappropriate antimicrobial prescribing in ambulatory practices. Infect Control Hosp Epidemiol 2018;39:307-15.

8. Brunton LL, Chabner BA, Knollmann BC. Principles of prescription order writing and patients compliance instructions. In: Brunton LL, editor. Goodman and Gilman's The Pharmacological Basis of Therapeutics. $12^{\text {th }}$ ed. New York: Pergamon Press Inc.; 2010. p. 1640.

9. Badar VA, Navale SB. Study of prescribing pattern of antimicrobial agents in medicine intensive care unit of a teaching hospital in central India. J Assoc Physicians India 2012;60:20-3.

10. Roberts AW, Visconti JA. The rational and irrational use of systemic antimicrobial drugs. Am J Hosp Pharm 1972;29:828-34.

11. Shankar PR, Partha P, Nagesh S. Prescribing patterns in medical outpatients. Int J Clin Pract 2002;56:549-51.

12. Patterson HR. The problems of audit and research. J R Coll Gen Pract 1986;36:196.

13. John LJ, Padmini D, John J, Goido S. Drug utilization study of antimicrobial agents in medical intensive care unit of a tertiary care hospital. Asian J Pharm Clin Res 2011;4:81-4.

14. Naikwadi IB, Baig MS, Bhattacharya M. To study the prescription pattern of antibiotics in medicine intensive care unit at tertiary care hospital. Int J Basic Clin Pharmacol 2019;8:738-45

15. Anand N, Nayak IM, Advaitha MV, Thaikattil NJ, Kantanavar KA, Anand S. Antimicrobial agents' utilization and cost pattern in an intensive care unit of a teaching hospital in South India. Indian J Crit Care Med 2016;20:274-9.

16. Perveen RA, Nasir M, Farha N, Islam MA. Antibiotics in ICU: The challenges of use, cost and response in a tertiary care hospital. Int J Med Res Health Sci 2018;7:94-9.

17. Smythe MA, Melendy S, Jahns B, Dmuchowski C. An exploratory analysis of medication utilization in a medical intensive care unit. Crit Care Med 1993;21:1319-23.

18. Srishyla MV, Krishnamurthy M, Nagarani MA, Mary CS, Andrade C, Venkataraman BV. Prescription audit in an Indian hospital setting using the DDD (defined daily dose) concept. Indian J Pharmacol 1994;26:23-8.

19. Mangrulkar SV, Mangrulkar S, Khair P, Phalke A. Antibiotic use in the 
intensive care unit. J Assoc Physicians India 2012;60:15-8.

20. Pandiamunian J, Somasundaram G. A study on prescribing pattern of anti microbial agents in the medical intensive care unit of a tertiary care teaching hospital in Puducherry union territory, South India. Int $\mathbf{J}$ Pharm Sci 2014;6:235-8

21. Nikhilesh A, Nagendra IM, Advaitha MV, Noble JT, Kiran A, Kantanavar KA, et al. Antimicrobial agents' utilization and cost pattern in an intensive care unit of a teaching hospital in South India. Indian J Crit Care Med 2016;20:274-9.

22. Shankar PR, Partha P, Dubey AK, Mishra P, Deshpande VY. Intensive care unit drug utilization in a teaching hospital in Nepal. Kathmandu Univ Med J (KUMJ) 2005;3:130-7.

23. Williams A, Mathai AS, Phillips AS. Antibiotic prescription patterns at admission into a tertiary level intensive care unit in Northern India. J Pharm Bioallied Sci 2011;3:531-6.

24. Alamchandani RR, Khubchandani PR. A study evaluating appropriateness of utilization pattern of antimicrobials in patients admitted in medical intensive care unit of tertiary care teaching rural hospital. Int J Pharm Pharm Sci 2015;7:101-4.

25. Viktil KK, Blix HS, Moger TA, Reikvam A. Polypharmacy as commonly defined is an indicator of limited value in the assessment of drug-related problems. Br J Clin Pharmacol 2007;63:187-95.

26. Kutty KV, Narmadha S, Nagarajan M. A study on drug prescribing pattern in Madurai city. Indian J Pharmacol 2002;34:361-2.

27. Curcio D. Antibiotic prescriptions in critically-ill patients: A Latin American experience. Ann Med Health Sci Res 2013;3:220-8.

28. Amit GS. Drug use evaluation study in a tertiary care corporate hospital with special reference to use of antibiotics in ICU department. Int J Adv Pharm Biol Chem 2013;2:179-89.

29. Admane PD, Hiware SK, Mahatme MS, Dudhgaonkar SD, Deshmukh SN, Mahajan MM. Prescription pattern of antimicrobials in tertiary care hospital in central India. Int J Pharmacol Res 2015;5:31-4.

30. Alharafsheh A, Alsheikh M, Ali S, Baraiki AA, Alharbi G, Alhabshi T, et al. A retrospective cross-sectional study of antibiotics prescribing patterns in admitted patients at a tertiary care setting in the KSA. Int $\mathrm{J}$ Health Sci 2018;12:67-71

31. Jana SK, Mondal P, Bhattacharya SK. A historical perspective on the rational use of drugs (RUD) in India. Indian J Pharmacol 2006;38:374-5.

32. Jokandan SS, Jha DK. A study of prescribing pattern of antibiotics in a tertiary care hospital-an observational study. Int J Pharm Sci Res 2019;10:2285-89.

33. Hanssens Y, Ismaeili BB, Kamha AA, Elshafie SS, Adheir FS, Saleh TM, et al. Antibiotic prescription pattern in a medical intensive care unit in Qatar. Saudi Med J 2005;26:1269-76.

34. Gowthami B, Spurthi T, Afreen SS. Drug utilization evaluation of antibiotics in general medicine department of a tertiary care hospital. Int J Pharm Pharm Sci 2016;8:302-4.

35. Usluer G, Ozgunes I, Leblebicioglu H. A multicenter point prevalence study: Antimicrobial prescription frequencies in hospitalized patients in Turkey. Ann Clin Microbiol Antimicrob 2005;4:16

36. Biswal S, Mishra P, Malhotra S, Puri GD, Pandhi P. Drug utilization pattern in the intensive care unit of a tertiary care hospital. J Clin Pharmacol 2006;46:945-51.

37. Shankar RP, Partha P, Shenoy NK, Easow JM, Brahmadathan KN. Prescribing patterns of antibiotics and sensitivity patterns of common microorganisms in the internal medicine ward of a teaching hospital in Western Nepal: A prospective study. Ann Clin Microbiol Antimicrob $2003 ; 2: 7$.

38. Maharani B, Jafrin AL, Prakash M, Priyadarshini P. Prescription pattern of patients admitted in the intensive care unit of a tertiary care hospital in Puducherry, India: A cross sectional study. Int J Basic Clin Pharmacol 2017;6:2822-26. 